\title{
Computed Tomography of the Chest without Contrast
}

National Cancer Institute

\section{Source}

National Cancer Institute. Computed Tomography of the Chest without Contrast. NCI

Thesaurus. Code C137898.

Computed tomography of the chest without the use of a contrast agent. 